\title{
Cognitive Effects of Late Life Depression: Review of Neuropsychological Findings
}

\author{
Maria Stylianou Korsnes, Ingun D. Ulstein \\ Department of Old Age Psychiatry, Oslo University Hospital, Ullevaal, Norway \\ Email: $\underline{\text { m.s.korsnes@psykologi.uio.no }}$
}

Received 6 February 2014; revised 10 March 2014; accepted 18 March 2014

Copyright (C 2014 by authors and Scientific Research Publishing Inc.

This work is licensed under the Creative Commons Attribution International License (CC BY). http://creativecommons.org/licenses/by/4.0/

(c) (1) Open Access

\begin{abstract}
Normal aging and depression both slow information processing speed which leads to poorer attentional control, shallower inhibition monitoring, poorer encoding, poorer updating of short term memory, and subsequent poorer learning, set-shifting, error monitoring, inhibition control, and cognitive planning. A combination of aging and depression amplifies these cognitive effects. Deterioration in frontal brain functioning in normal aging is irreversible, but altering of frontal brain function due to depression may be reversible. Since the symptoms are indistinguishable, it is difficult to separate effects of depression and aging with use of neuropsychological tests, even when premorbid history is known. Statistical group effects show more severe cognitive effects in late life depression than in normal aging or depression in younger persons, but no particular cognitive markers of depression in older subjects have been identified. Attention and inhibition are closely related, but their relationship needs to be studied further. It is not clear, whether a problem with inhibition of irrelevant stimuli occurs because of attentional deficits due to late life depression, or if it is a primary consequence of the depression. Further studies on the relationship between inhibition, attention and processing speed involving homogeneous depressed and nondepressed groups of different age may help disentangle this relationship.
\end{abstract}

\section{Keywords}

Neuropsychology; Cognitive Testing; Aging; Depression; Information Processing; Inhibition; Attention; Cognition

\section{Introduction}

Previous literature has suggested that cognitive functions are affected in depression and that cognitive decline due to depression may be permanent or lasting, even when the patient enters a euthymic stage [1]. Despite the 
increasing attention on this topic, much is still unknown regarding how cognition is affected by depression [2]. Particularly, it is not known to what extent severity of depression causes nonlinear effects on cognition. Secondly, it is not clear which aspects within each cognitive domain that is caused by depression, and thirdly the core underlying factors leading to decline within each cognitive domain may be different [2]. In order to clarify these issues, cognitive sub-function within each cognitive domain needs to be independently investigated, and great care should be taken before concluding on the presence or absence of group differences within a particular cognitive domain. The bulk of literature assessing cognitive functioning makes use of standardized neuropsychological tests, which is a great advantage due to general availability and reliability of results across populations and labs. However, standardized neuropsychological tests are not designed for benchmarking particular cognitive sub-functions between groups, since a particular test often tap into several sub-functions within one or several cognitive domains.

Since each cognitive function most often depends on other cognitive functions, it is not easy to pinpoint which function is primarily affected by the depressed state. In several earlier reports these functions have been merged together, and new definitions have been created to fit the data. For example, Story et al. [3] included attention and processing speed in their definition of executive functions, since these functions also rely on prefrontal and subcortical brain-structures. However, this approach makes it difficult to assess which of the underlying cognitive sub-functions are directly affected by the depressed state and which are affected because of dependencies of interconnected cognitive sub-functions. Several scientific papers concluding on deficits in certain cognitive functions due to depression may not have controlled for the underlying processes. Tests may actually show deficits in other cognitive domains than assumed by the researchers, or may reflect poor planning and executing of the task due to problems related to basic cognitive abilities, such as attention to the stimuli, speed of processing or motor function.

In the present paper, we review the current literature with focus on practical application rather than theoretical framework, and investigate which cognitive sub-functions are directly or indirectly affected by depression. The review particularly focuses on the cognitive effects in late life depression, where cognitive deficits are more severe than in younger adults [4] [5]. Studies were selected from the Pubmed Medline, PsychInfo, Embase and Cochrane Databases. All reviewed papers were written in English, and were published between 1980 and 2014. Search terms were "depression” or "depressed” or "depressive” or "MDD” AND “aging” or "ageing” or "geriatric" or "late life" or "old age" or "late onset" or "elderly" or "older" AND "neuropsychological" or "neuropsychology" or "cognitive" or "cognition" or "memory" or "executive function" or "attention" or "information processing" or "encoding” or "learning or "inhibition". The search was repeated without the aging terms in order to identify the most referred articles on neuropsychological findings on depression.

\section{Cognitive Neural Networks}

Cognitive decline has brain related correlates that may be measured with brain imaging techniques, such as fMRI. Neuroimaging studies have suggested that brain activation related correlates of depression most often are found within a frontal network that is associated with cognition in general, and particularly with attentional ability and executive function. There are several brain connectivity networks that correspond to attentional functions, such as a network corresponding to attention during visual orientation [6], a network of effortful/sustained attention [7] [8], an executive network corresponding to shifts in attention [9]-[12] and a network of attention related to stimulus inhibition [13]. These brain connectivity networks that in particular have been proposed following results of imaging studies, define a frontoparietal attention network (FPAN) of the human brain [6]. This network is thought to rely on an attention system where regions such as the Intraparietal sulcus (IPS) defines the priority for the attention network, and where the dorslolate prefrontal cortex (DLPFC)/dorsal anterior cingulate cortex (dACC) play important roles for keeping attention to the proper task in question. Interrupts may be generated in several prefrontal/premotor areas (PFC/PMC) including the frontal eye fields (FEF) for visual information where the inferior parietal lobe (IPL) is important for shifting attention if required, causing the IPS's "attention map" to be changed, either temporarily or permanently [6] [9].

There are also several other specific brain regions which have been associated with cognitive decline due to depression. These are limbic areas including the amygdala, the medial temporal lobes and other cortical and subcortical areas [4] [14] [15] or regions and networks related to affection and inhibition control, such as the pregenual/subgenual ACC, orbitofrontal area (BA47), and the posterior cingulate cortex (PCC) [13] [16] [17]. Integrity of the rostal anterior cingulate cortex (rACC) has been found to be important for cognitive control, in- 
cluding response inhibition when conflicts are being detected and during subsequent evaluation of errors [18] (Figure 1).

\section{Cognitive Functions in Late Life Depression}

Depression is one of the most common mental disorders, and is especially prevalent in the elderly, particularly elderly who suffer from a chronic medical condition or cognitive impairment [5]. It is important to consider whether depression in aging may be a warning sign of cognitive decline. In fact, depression and dementia often go hand in hand, and patients with dementia and depression must be grouped separately. Memory deficits in elderly may thus reflect early signs of neurodegenerative disorders in some patients [4] due to deficits in specific regions or connections between them, and cognitive changes may be early warning signs for the development of Alzheimers disease (AD) or other dementias. It is possible that some of the depressed elderly already suffer from brain impairment which may be associated with neuronal decline or injury, and develop depression because of the underlying degenerative factors or vascular brain lesions, and not necessarily reflect the depressed state they are in [4] [14] [15]. Presence of cortical dementia is thus an important variable that needs to be controlled for in order to assess the cognitive effect of depression in the normal aging population.

\section{Information Processing Speed}

Several researchers have suggested that almost all cognitive effects of aging are secondary to impairment in motor slowing and subsequent slowing in information processing speed [19] [20]. Correspondingly, slowing in information processing speed is one of the most reported findings of depression, making the effects of depression in the elderly difficult to distinguish from normal age related decline [14] [21] [22]. McClintock et al. [2] found in their review that depressed patients often do poorly on tests that are timed, which affects scores on information processing scales. Butters et al. [21] pointed out that slower processing speed in depressed patients in turn affect a range of other cognitive functions which rely on sufficient time for attending to stimuli which subsequently is required for effective encoding of rapidly occurring information. Decline on cognitive tests within several domains correlate with a similar decline in information processing speed, even when relevant covariates

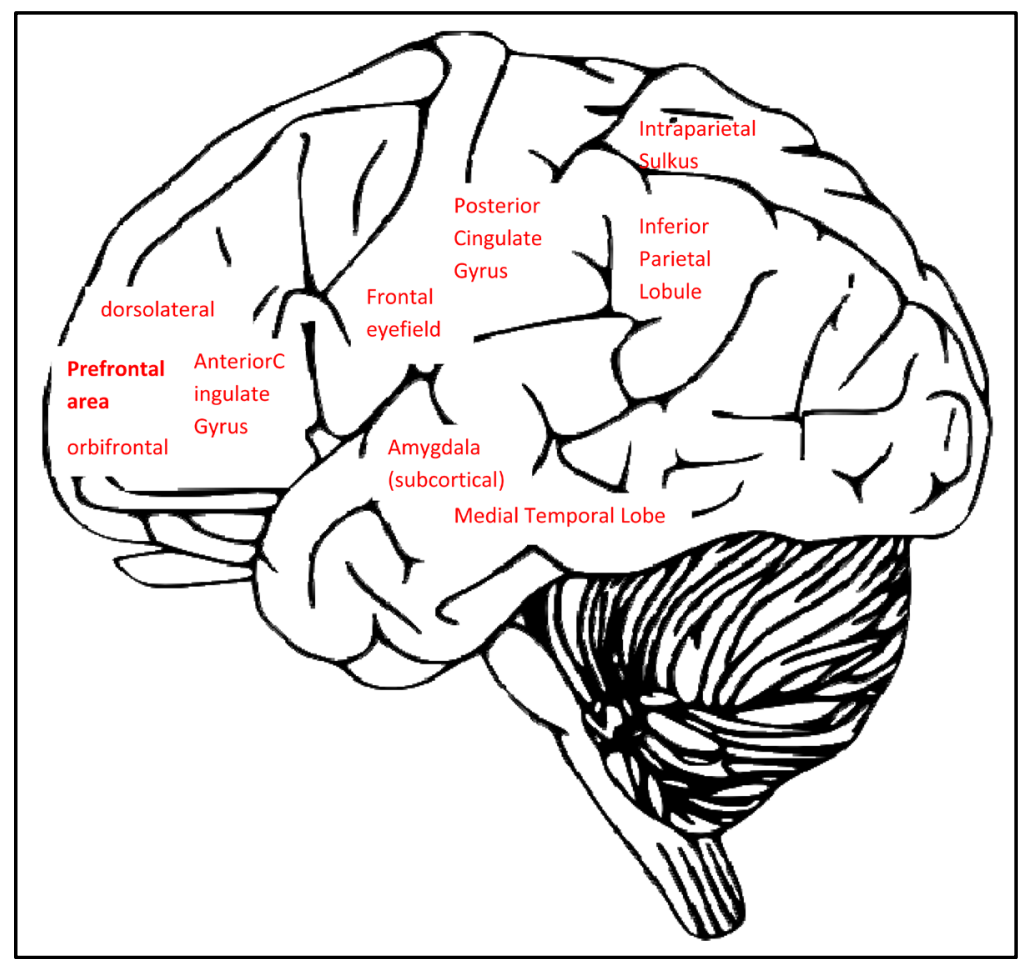

Figure 1. Brain areas related to cognitive effects of depression (brain image with permission from Clker.com). 
such as education and age is accounted for [23] [24]. Koster et al. [25] suggested a model where delayed and shallow processing of information is due to an inability to disengage from the negative self-referent processes driven by depression.

\section{Attention}

Attention is a multifaceted concept that is linked to concentration [22] [26], processing speed [22] [27] [28], executive function [3] [5] [29]-[31], motor function [19] [20], memory [4], and language [32]. Depression affects attentional ability that in turn affects these related cognitive functions [2]. Since general interest for external impulses often is reduced in depression, it is intuitive that attention will be reduced in depression, but it is not clear from previous reports whether this is true for all aspects of attention. Antidepressant medication may influence attentional performance, and is an additional variable that should be controlled for in experiments involving attention. Attentional processes depend upon frontoparietal structures that also are central for executive functioning [6]. Most attentional tests require motor feedback and are measured in response accuracy and speed of processing [19] [20].

\subsection{Automatic versus Controlled Attention}

Attention is often an automatic process related to the monitoring and awareness of the world around us [33]. Automatic versus effortful and controlled attention is also an integral concept of a theoretical framework called "two-process theory of attention", which makes a distinction between a focused attention mode when identifying targets and a diffuse attention mode when scanning non-targets in a sustained attention task. In the two-process theory of attention, rapid automatic attention scanning goes on in parallel with other processing, and generates interrupts when a target is identified, while controlled processing is a slower and exclusively serial short term memory process. However, when a person is in a focused state of attention, the person is less receptive to these automatic interrupts [34]. The automatic and controlled processes are assumed separate processes that may occur simultaneously, and depend on interlinked but separate brain processes. Attention and short term memory capacity in depression has been found to primarily be associated with controlled, focused or effortful processing [35], similarly short-term memory retrieval in effortful attentional processing is also impaired in depression [8] [36] [37] while automatic processing only are affected under severe conditions [38].

\subsection{Sustained Attention}

Sustained attention or vigilance requires a combination of both automatic and controlled processing depending upon the task [39]. Decline in ability to sustain attention may reflect dependency upon work-load intensity factors and results may vary between tasks. Sustained attention is important both for simple routine tasks where losing attention could lead to mistakes, as well as more difficult specialized tasks which require a higher level of concentration. One frequently used test is the Trail-making test A (TMT-A), which comes in several versions [31] [40] [41], e.g. from the Halsted-Reitan battery [42]. The TMT-A requires the participant to draw a trail between numbers on a sheet of paper and requires several additional cognitive abilities, such as information processing, sequencing ability, and motor speed. Thus, it is important that these other cognitive factors must be controlled for in a group analysis. There are several tailor made tests available that measures sustained attention, one is the Continuous Performance Test (CPT, [43]) which is available in both auditory and visually presented versions. The CPT requires response to a non-frequent target letter every time it appears in a stream of letters, but no response to frequent non-target letters. Another similar test is the Sustained Attention to Response Task (SART test; [27] [44]). In the SART test, the subjects are required to restrain from responding to a fraction of the targets, while they are required to respond to the rest of the targets, and accuracy and response times are measured. This test show poorer sustained attention even in moderately depressed men [27]. However, there is still not conclusive evidence to claim that sustained attention decline as a function of aging [45] or as a function of depression [2].

It has long been known that arousal is an important component in order to facilitate sustained attention to stimuli [46], and arousal depend upon subcortical brain regions including the amygdala that also show deviating activation results for depressed versus normal subjects in brain imaging studies. One prefrontal region, the cholinergic system, releases acetylcholine under normal operation. It has been shown that blocking release of ace- 
tylcholine impairs the ability of sustained attention [47]. In addition, rise in acetylcholine level has been found to invoke more severe depression, implicating normal cholinergic release as important in depression and other affective disorders [48].

\subsection{Selective Attention: Focused or Divided Attention Mode}

The typical example in order to describe the divided attention situation is a cocktail party [49], where several conversations may go on in parallel within hearing distance, and it is possible to tune in to one particular conversation while registering bits and pieces from other conversations. Attention may be switched if something interesting comes up in any conversation within hearing distance. In the visual mode, divided attention refers to changing viewpoint, e.g., focusing towards movement in the peripheral field of view when driving a car. In the dichotic listening test, consonant-vowel syllables are presented in headphones to both ears, and the participant are asked either to attend to stimuli from a particular ear, or to attend to stimuli without a reference to lateralization of the input. A right ear preference is found in normal adults, however the right ear advantage (REA) is altered in several patient groups, including in schizophrenia where lack of REA is interacting with the occurrence of auditory hallucinations [50]. A similar pattern of missing asymmetry in dichotic listening tests has been reported in major depression, particularly for patients with withdrawal-retardation symptomatology [51]. It is possible that the combination of the affective and attentional reductions due to depression contributed to lower accuracy on the task in the depressed group as compared to the control group, and this may be related to a lack in top-down control of the cognitive resources, including the resources required for maintaining attention to tasks or stimuli.

Divided visual field tasks have shown reduced visual divided attention in MCI patients and Alzheimer patients [52], and a similar pattern of reduced divided attention may be predicted in depression, since depressed patients may be lacking the ability of focusing visual attention towards the appropriate spatial position of the target, based upon the assumption of a similar top-down control deficit in depression for the visual mode, as was suggested for the auditory mode. Hammar et al. [53] found effortful processing to be reduced in two testing sessions despite improved scores on a depression rating scale (HAM-D; [54]) between sessions when performing a visual search paradigm. All patients had a history of recurrent depression. In addition, Majer et al. [55] found that performance on a dual auditory / visual divided attention task (part of "Test batterie zur Aufmerk-samkeitsprüfung”, "TAP”) was reduced in depressed patients, suggesting that depression causes deficits in divided attentional performance regardless of perceptual mode. They found a direct correlation between divided attention task performance and depression. The study controlled for medication, depression severity (depression rating score) and depression duration.

\section{Memory and Learning}

Memory processes, such as episodic memory are found to be impaired in depression, particularly in elderly depressed [2] [56]. Elderkin-Thompson et al. [56] concluded that memory and learning deficits in depressed elderly correlated with activation effects in frontal-striatal pathways, and suggested that underlying executive dysfunction resulted in cognitive decline in memory and learning. These brain regions are also essential for attentional processing [6]. In order to assess the underlying causes, the component processes leading up to memory and learning needs to be assessed individually.

\subsection{Encoding and Retrieval}

If a person is preoccupied with depressive thoughts, the threshold for registering a new event is elevated. This leads to interrupts in the processes leading up to memory formations, with shallow encoding of events, and lesser formation of enduring memories [21] [57]. Since processing speed is one function that naturally is showed in aging, memory deficits as a consequence of depression tend to be even more severe in elderly, due to even shallower encoding of new events [57] [58]. Episodic memory impairment in depression is associated with shallow encoding and poorer effortful control of encoded information [59]. Baune et al. [22] performed a principal components analysis in order to extract the memory components from several tests on elderly, and they did not report significant effects on episodic memory due to depression when other effects were controlled for. Thus, 
memory problems in depression are at least partly, if not exclusively based on other underlying cognitive functions.

The ability to "remember" implies a controlled recollection or recognition of the encoding event or study episode, while "know" reflect a more automatic and effortless processing. "Remember" and "know" responses reflect two recollection mechanisms which have been found to be functionally independent in that remembering depend more on perceptual factors and knowing depend more on conceptual factors even though there are overlaps [60] [61]. Experiments with the remember-know paradigm show reduced ability of making "remember" responses in normal aging while "know" responses are unaffected [62]. Yonelinas et al. [62] found an association between less elaborative encoding and reduced remembering, and they suggested that older participants were less able to use the appropriate contextual retrieval processes. A similar pattern was found in major depression [63], suggesting again that the effect of impaired recognition memory should be even stronger in late life depression, due to impairment in recollection processing rather than familiarity processing. The underlying factors may be shallow encoding due to slowed information processing, but it is also possible that independent factors that affect strategic memory planning or other executive planning are affected in depression. Since independent aging [62] and depression studies [63] show similar pattern of results using the remember/know paradigm, it may be assumed that aging and depression affect the same underlying factors.

\subsection{Updating of Short Term Memory}

Updating of short term memory is an information processing function that relies on sufficient processing speed. It is often treated as an executive sub-function or as an attentional control function, since it involves an active strategy in order to focus attention, refreshing, and monitoring what is contained in the limited working memory [64]. Updating of working memory has been studied with n-back go/no-go tasks where the participant decides whether a letter is the same as the one presented $n$ letter earlier in a list (e.g., [24]), or with serial list recall tasks with verbal or spatial stimuli [12]. Updating of short term memory has been found to be impaired in both depression and in normal aging, and thus updating of short term memory is particularly impaired in late life depression [24]. However, since updating of short-term memory depends on processing speed and attentional control, impairment on short-term memory task are likely to reflect impairment in these sub-functions.

\subsection{Learning}

It has been reported specific effects on verbal memory in late life depression, where recalling list learning items is affected while recalling storytelling is unaffected [65]. Lamar et al. [65] compared brain area volumes in late life depression with matched healthy controls, and found a correlation between volume sizes of prefrontal and cingulate sub-regions with poor performance on the list-learning task (California Verbal Learning test, CVLT) in the late life depression group. These brain areas are important for all cognitive functioning. The study show a dissociation between list-learning performance and recollection of storytelling analogous with the dissociation between remembered and known responses in the remember/know paradigm. One common factor is that more structured and effortful encoding paradigms influence subsequent recollection more than it influence automatic or less structured encoding paradigms. In the Lamar et al. study [65] the mini-mental state exam (MMSE) did not show any group differences, indicating that the groups do not differ on brain organic state, and thus that result in that study reflects a connection between brain area size and depression score. Cause-effect connections should be explored further, and more detailed regional divisions should be considered.

Learning relies heavily on strategic and executive skills, including mental flexibility and perceptual organization [2], skills that require alertness and attentional abilities. Older adults, regardless of mental state, are less capable of meta cognitive control over their own learning, since older adults have been demonstrated to be much less effective in restudying items by choice [66] again demonstrating less effective information processing in older adults. Thus, it should be expected that learning ability should be more impaired in older depressed than in younger depressed. Indeed, in their meta study McClintock et al. [2] refer studies which have found significantly higher error rates in late life depression than in non-depressed age matched controls on several learning tests, including the Cambridge Neuropsychological Test Automated Battery (subtest on Visuospatial Paired-Associates Learning), Rey Auditory Verbal Learning Test (RAVLT, verbal list-learning and distractor list), and the Hopkins Verbal Learning Test and the California Verbal Learning Test (CVLT). 


\section{Executive Function}

The term "executive function" is an umbrella term which refers to a number of cognitive processes involved in regulation of thought, planning and action. These high-order processes may either be triggered by bottom-up interrupts, or be goal-directed top-down processes. These functions may have common or interconnected neural correlates, but since each subdomain may rely on processing in different sub-regions or on different connections between brain areas, tests of the "executive function" are not very specific but can be useful since generally reduced executive functioning capacity in turn affect other cognitive domains such as social control [12] [19] [21] [22]. One common denominator for functions within the "executive" umbrella is that they all rely on frontal brain areas, since patients with lesions to frontal brain structures, including the dorsolateral frontal lobe, perform poorly on tests that require set-shifting, strategic planning, searching, and utilizing feedback for task performance [67].

One test that measures several executive functions simultaneously is the Wisconsin Card Sorting Test (WCST; [68]), in which the patient is presented with cards of different color, symbols and number of symbols. Their task is to match a card with several other cards. The patient is not told specifically whether to match for color, symbol or number of symbols but he or she is given a "correct" or "wrong" feedback. After a while the participants sorts cards according to different rules, such as number of symbols on the cards, color, or symbol type, and the patient must then be able to inhibit using the previous rule, figure out the new rule (updating of short-term memory), and shift sets (reactive flexibility) in order to solve the new task. The WCST also relies on the integrity of other high level cognitive functions, such as preservation and abstract thinking. Even though processes such as inhibition, updating, and reactive flexibility are interlinked, they are independent cognitive processes [69]. Thus it is often necessary to administer tests of each executive subdomain separately, particularly in aging research, since normal aging may affect these subdomains differently. In a comprehensive study with three tests on each subdomain, Albinet et al. [12] found that age predicted approximately $42 \%$ of the variance in set-shifting, $31 \%$ of the variance in inhibition, and $25 \%$ of the variance in updating of working memory. In their study, they compared a young and an older group matched for education.

\subsection{Set Shifting Ability}

Set shifting ability is consistently found to be reduced in affective disorders, including eating disorder [70] [71], obsessive compulsive disorder [72] and depression [21]. Set shifting and sustained attention to new stimuli are interdependable cognitive processes [32], and it is challenging to measure "attention" and "set-shifting” separate from each other. Set shifting is often measured with the trails-B test (TMT-B, drawing a line between alternating numbers and letters); [42] and/or the WCST (which rely on several closely linked executive functions, see above), but interpretations are not straight forwards. Impairments on the TMT-B in depressed groups may be best explained by the attentional aspects of that task [30]. Butters et al. [21] found that all cognitive effects in late life depression, including the TMT and WCST were mediated by slowed information processing speed, which may also be seen as an indicator for an underlying attentional deficit in depression. The Stroop test [73] consists of three tasks, either to judge the color of stimuli, read color words, or read color words written in a different color than the color mentioned in the text. This latter test is labeled the Stroop interference test. In the study by Butters et al. [21], late life depressed patients were impaired on both the TMT-B and WCST when compared with a control group, but not on the Stroop test which primarily is a measure of cognitive flexibility, sustained attention and inhibition, since it measures interference between tasks rather than shifting between them.

\subsection{Spontaneous Flexibility and Verbal Fluency}

Spontaneous flexibility requires an active search for alternatives rather than a reactive adjustment to changing rules. One example is the ability to create multiple words according to specific rules within a limited time, as in the F-A-S test [74]. This test requires the participant to find as many nouns as possible starting with the letter F, A, and S. Performance on this task relies heavily on frontal brain structures, since patients with frontal lobe related deficits often perform poorly on word generation on one or all letters. The ability to actively create words depends on motivation and alertness. Thus, low FAS score correlates with increasing apathy in dementia [75] but does not correlate with apathy in depression [76] even though verbal fluency is often referred to as reduced 
in depression [2]. The FAS-test and other verbal fluency tests used to measure spontaneous flexibility and verbal fluency all rely on intact processing speed and attentional ability, thus factors related to attention and processing speed may be the principle components for low results of the FAS-test in depression [2] [25]. In group studies, it is important to control for these other factors which may lead to reduced verbal fluency or spontaneous flexibility on the individual level.

\subsection{Source-Monitoring}

Source monitoring imply recollecting how we obtained one piece of information, and may involve different processes depending on whether the source is known to be self-generated or externally generated, or whether it is unknown if the source is self-generated or externally generated [77]. Source monitoring of either self-generated or external sources has been found to be poorer in older than for younger adults [24]. Older adults perform particularly poorer when discriminating between externally generated sources as opposed to self-generated sources [78]. Performance in elderly correlated on a measure of inhibition (Stroop-C interference task)/(Strop A $+\mathrm{B})$ ), pointing to poorer inhibition control in elderly as one reason for poorer source monitoring. Another possible explanation for these results is that the more complex interference task (C) require more than the available information processing power than the simple Stroop tasks (A and B), and that the results reflect poorer information processing ability in the older subjects. Source monitoring deficits have also been reported in depression. Ulrich and Meiser [79] found that depressed patients when assessed with the Beck Depression Inventory [80] showed better memory for irrelevant stimuli relative to stimuli required for task performance in a source monitoring experiment. The subjects had to decide if a word was old or new, and if old, if the word had been presented on the left or the right side of the screen, and if the word had been presented inside a green frame or a red frame. Even though the depressed patients performed comparable to the control group on the source monitoring task, the depressed group paid significantly more attention to stimuli that were irrelevant to the task completion, suggesting that defocusing of attentional resources in depression may be important for cognitive effects of depression.

\subsection{Conflict Control and Error Monitoring}

Awareness of making errors is important for performance enhancement and learning to perform a task. When humans commit errors, even when not explicitly aware of it, a massive negative peek can be measured from central and frontal electroence phalography electrodes in experiments tracing event related potentials. The negative peak has been labeled error-related negativity (ERN). Functional Magnetic Resonance Imaging (fMRI) evidence points to the origin of this peak within the anterior cingulate cortex (ACC) in particular [81] [82] and also in the dorsolateral prefrontal cortex (DLPFC) to some degree [13] [83]. The response may be a subconscious response to simple detection of errors, or perhaps to a more complex conflict monitoring system [84].

Performance monitoring is essential in order to solve certain complex tasks, such as the Stroop interference test. Patients with major depressive disorder (MDD) perform poorly on this type of task. Holms \& Pizzagalli [85] reported reduced ERN-peaks in N2 and N450 amplitudes in patients with major depression when making errors, thought to originate ACC and the left DLPFC. The MDD patients that performed poorest on the Stroop interference test exhibited the lowest ERN-peak in dACC following stimulus presentation, indicating that poor conflict monitoring may correspond to poor performance on the Stroop interference test. The cause of the low dACC activation may also be related to conflicting processes related to the depression, which perhaps rely on the dACC or interconnected brain areas. Recent studies have shown that ERN-peak correlate with a wide range of affective and emotional factors [86] [87].

Georgiadi et al. [88] used a go/no-go task to compare remitted major depressive patients and acutely depressed patients with controls. They reported significantly higher ERN peaks in the remitted patients, and nonsignificant ERN-peak reduction in acutely depressed. They also observed a trend of decreased ERN-peaks with age in all study groups, suggesting that ERN-peak normally is reduced with age, corresponding to reduction in acutely depressed patients as compared to remitted patients. These ERN results suggest that regions within the ACC corresponding to an error monitoring system are abnormally active in remitted depressed patients, while several factors, including aging and acute depression reduces activation in the error monitoring system. The higher ERP-peaks in remitted depressed patients may indicate that depressive periods can cause some permanent effects to the ACC [1], but it is also possible that these effects were pre-existing in the remitted depressed pa- 
tients. In that case highly activated ERN-peaks may be an indicator for risk of developing depression.

\subsection{Inhibition Control}

The ability to control actions and stop inappropriate or erroneous responses is important for a wide range of situations and crucial for efficient task performance and mental processing [13] [89]. Inhibition is not one process, but several conscious and unconscious processes that suppress the irrelevant information and enables focusing and concentration on an important task. Inhibition performance is commonly tapped by the Stroop interference task, but also with several task-switching paradigms, semantic generation tasks with rules which require response inhibition, random number generation which require response monitoring and inhibition of repetition of numbers, the stop signal task (SST) where the participant responds to rapidly presented stimuli but withhold the response when a simultaneous tone is presented, or tasks where the participant must inhibit the logical response. For example Tap 2 from Luria and Homskaya [90] where the participant were asked to tap two times when the experimenter shows them one finger and to tap one time when the experimenter shows them two fingers.

Reduced performance on inhibition control tasks is strongly associated with depression [18] [58] [91]-[94], and inhibition and inhibition control may be key factors to our understanding of depression. Age is also a contributing factor to reduced inhibition control, and age has been found to predict $30.8 \%$ of the variance in inhibition when level of education was controlled for [12]. Thus, it may be assumed that inhibition control is of particular importance in late life depression. De Lissnyder et al. [94] found that depressed patients with rumination symptoms were significantly more impaired in executive control tasks than depressed patients without rumination, suggesting that symptomatology and depression severity should be controlled for in order to assess the role of inhibition control in depression.

Animal research has shown that certain neurotransmitters i.e., acetylcholine may trigger the ACC to enhance inhibition of the lateral prefrontal cortex [95], and human research indicate that increasing cholinergic activity also may shift human behavior towards inhibition and poorer cognitive control in manic depression and schizophrenia [48]. Integrity of the rostal ACC has been found to be important for cognitive control, including response inhibition when conflicts are being detected and during subsequent evaluation of errors [13] [18]. The functioning of the rostal ACC has been found to be altered due to depression, as shown by deviating ERP responses in depressed older adults following an emotional go—no/go paradigm [93]. Also, smaller gray matter volumes of the ACC correlated with poor effects of antidepressant treatment [96], indicating that preexisting conditions affecting the anterior cingulate may be one reason why many elderly depressed patients do not respond to common antidepressant treatments [18]. Reduced N2 components within ACC correlate with poorer performance on the inhibition control task, suggesting that depression causes less efficient processing within the ACC, or perhaps that brain plasticity has shifted inhibition control processing to less specialized areas [13] [18]. However, it is not clear whether the mechanisms behind the altered functioning of the ACC may be a direct consequence of depression, or if a deficit within the ACC causes depression.

Katz et al. [18] found that deficit in inhibition control vary as a function of task load. Their data suggested that ERP responses were normal in depressed subjects, but abnormal peaks originating from the ACC occurred in depression patients under high-load conditions only. ERPs which normally show a peak of the N2 component during successful inhibition control is reduced in depressed elderly, accompanied by enhanced N2 components in the middle frontal gyrus [18]. Thus, underlying factors, such as limited capacity in processing due to poorer information processing speed and subsequent attentional factors must be important also for inhibition and inhibition control, since inhibition control is poorer under high task load.

\subsection{Self-Organization and Cognitive Planning}

Deficits involving planning may be caused by deficits in a number of underlying functions, such as the ability to attend to a task or insufficient speed of processing. It is clear from previous research that high level executive functioning such as planning particularly relies on the integrity of prefrontal brain regions that also are important for attention, such as the dorsolateral frontal lobe [6].

Cognitive planning may be assessed with the "Zoo Map Test” which consists of two subtests, one low demanding test where the participants only follow a predefined strategy in order to proceed from one site to the next site in a zoo-map, and one high demanding test where the participants must plan their route according to complex rules [97]. Allain et al. [98] concluded that planning ability is affected in aging when comparing a 
young and an older age group using the "Zoo Map Test”. They found that the older group exhibited more problems than the younger group when they needed to plan a route than when they just followed a predefined pattern, and the authors suggested that elderly participants may have problems in developing "logical strategies". Since the "Zoo Map Test" also correlate with processing time, it is possible that group differences in processing time contributes to this finding. Oosterman et al. [99] compared this test against a number of other neuropsychological tests grouped in the domains of "planning", "memory", "inhibition" and "processing time" and found the strongest correlation for the "planning" domain. However, the authors note that processing speed also correlate with performance and that processing speed may be the strongest predictor of the "Zoo Map Test" when comparing different patient groups or less homogeneous groups.

Elderkin-Thompson et al. [56] used the "Rey Osterrieth Complex Figure test (ROCF)" when comparing a group of late life depressed with a control group, in order to assess whether poor performance on the in late life depression depends on reduced planning ability or reduced visuospatial ability. They found that poorer scores for a depressed group was quantified by planning simple copying of the ROCF figure and concluded that planning may be the underlying source of declining nonverbal memory in late life depression. Their results also shows that deviation on ROCF-copy scores in late life depression is disassociated from visuospatial ability, but it is possible that the decline in ROCF-copy performance reflect underlying poorer short term memory span due to poorer processing speed and/or attentional ability rather than planning ability.

\section{Discussion}

This literature review points to information processing speed as the common factor that may explain most of the cognitive decline found in depression, and depression in old age in particular, since normal aging also causes slower information processing speed. Information processing in depression is linked to slowing due to inattention, or perhaps preoccupation with negative thoughts [25], or a network of attention related to stimulus inhibition [13]. Slow information speed leads to poorer attentional control which in turn is vital for controlling and monitoring inhibition, shifting mental sets and encoding of working memory [64] [72] [100]. Poorer encoding of stimuli in turn leads to poorer updating of short term memory, and subsequent poorer learning, set-shifting, error monitoring, inhibition control, and cognitive planning. A very important component in this framework is that slowing in information processing affect the ability to inhibit attention to stimuli that are irrelevant for the focused attention. The neuronal correlates found in depressed groups most often overlap regions associated with an attentional network (see [6]). It is also clear from previous research that more severe depression, higher number of depressive episodes and longer lasting depression leads to longer lasting and more severe cognitive effects [2]. Attentional difficulties are more severe in ageing since depression symptoms overlap the common effects of normal aging [5] [21]. Thus, it is possible that most of the variation found within these domains in depressed patients is due to the chain of dependencies outlined here (see also [2]). This literature review finds no strong evidence for a direct link between depression and memory, learning or executive functions.

Arousal to the relevant stimuli is one factor that may boost sustained attention to relevant stimuli and inhibition of irrelevant stimuli, and the regulation of neurotransmitters in the cholinergic system that trigger appropriate activity in the ACC may be important regulators of appropriate arousal levels [95]. Increased cholinergic activity may cause inhibition of relevant stimuli and more severe depression in humans [48], while blocking of cholinergic activity impairs the ability of sustained attention [47]. It is unclear how these mechanisms interact with information processing speed.

There are studies that have identified lasting effects of depression in euthymic patients [1] which suggest that depression may alter the brain permanently. As depression affect brain function in areas involved in attention and executive control, at least on a temporary basis [6] it may be that this changing of brain activity pattern can lead to a permanent or lasting effects on cognitive functioning. However, more studies are needed in order to confirm if periodic depression may lead to permanent brain damage.

\subsection{Co-Factors}

Aging is an important co-factor which leads to more severe cognitive effects due to depression, thus age related decline in motor functioning, accuracy and speed of processing must be controlled for in group studies [19] [20]. Educational level is another factor that influences cognitive function, and thus most neuropsycological tests controls for these factors. In group studies involving elderly patients, the motor speed components should be 
controlled for with additional similar tests which do not require the same cognitive functions that are being tested. Also, as reviewed here, several other factors should ideally be controlled for in future studies of the cognitive effect on depression, which may lead to the requirement of larger sample groups than planned for initially. A principal components analysis (e.g. [22]) is one way of controlling for co-factors.

Depression posing as dementia is common in elderly individuals, presenting with symptoms of a recent cognitive decline. In contrast to Alzheimer's disease where short-term memory loss occurs as a cardinal first sign, all memory is affected equally in the depressed individual. Comorbid depression and degenerative conditions such as mild cognitive impairment and dementia may cause worsening of the symptoms of the degenerative condition or depression, but may be caused by different mechanisms, since faster than expected reaction times have been shown to cause poor performance on accuracy during information processing in dementia [44]. If depression and dementia are coexisting states, the time of onset of depression may help to pinpoint the etiology of the disorder. The Folstein Mini-Mental State Examination (MMSE) or the Montreal Cognitive Assessment (MoCA) are useful tools used for screening of cognitive deficits. The examiner will also look for signs concerning motivation and sense of reality. Patients with dementia tend to try to answer questions, but often fail, while depressed patients may show passivity and give "don't know" answers. When interviewing the patients, demented patients may be unaware, or deny having memory problems, while depressed elderly may complain about having memory problems.

Depression state and severity of depression should be controlled for in all group studies, since some cognitive effects may only be measurable in particular depression samples. Some effects of depression, e.g. inhibition control may be correlated to depression severity. In order to make a diagnosis of major depression, DSM-IV and/or ICD-10 definitions for the diagnosis must be met, including depressed mood or loss of interest or pleasure. Severity of depression may be measured on the Montgomery Aasberg Depression Rating Scale (MADRS, [101]), Beck depression inventory [80], the Hamilton rating scale for depression [54], or other Depression Rating Scales. There are several Depression Scales that have been evaluated for several age groups, e.g. the MADRS that has been shown to be valid screening instrument also for depression in elderly patients [102].

Antidepressant medication may influence cognition in several ways, since some medication may lead to additional slowing due to drowsiness, while eliminating symptoms of depression. Most antidepressant medication will affect alertness and other attentional properties. Thus, care should be taken before comparing medicated and non-medicated groups, and in particular mixed groups with different set of medication.

Hormonal differences between the sexes make it about twice as likely for women than men to develop depression, and the effects of depression may differ in women and in men. Thus, study groups should be balanced between sexes. Some other genic factors may be more difficult to control for in group studies, but it is possible that some genetic predispositions to depression may play a role in the development of cognitive impairment secondary to depression, but the evidence for this is weak [2]. Particular interest has been invested in the ApoE4 allele gene, which is known as a risk factor for Alzheimer's disease. Butters et al. [21] did not find any cognitive effects, particularly no effects on memory function in depressed elderly with ApoE4, hence there is no known link between the presence of depression and this gene.

Comorbidity with another affective disease with overlapping symptoms may lead to more severe cognitive effects on depression. Comorbid depression and anxiety is particularly common among elderly. In a Norwegian population based study including more than 1300 elderly, $9 \%$ had depression, $13 \%$ had anxiety, and 5\% had both [103]. This overlap should be controlled for, since cognitive effects of anxiety and depression both affect executive speed of processing, language, executive functions and memory functions [2] [104]. Also, both depression and anxiety disorders affect attention, although the cognitive effects are different. In depression, general alertness is biased due to a depressive state, while in anxiety disorders attention is biased towards situations which feels threatening. Little is known about the neural underpinnings that separate anxiety from depression, thus future studies are needed to address this relationship.

Comorbidity of depression and certain somatic conditions, such as vascular disease or cardiovascular risk factors (hypertension, diabetes, coronary artery problems) has a similar effect on cognition [105]. The common denominator is that comorbidity of any of these conditions leads to more severe degradation of the cognitive functions when compared with another depressed group without these comorbid conditions. It is possible that some of these conditions, just like aging, lead to slowing of processing speed, and might lead to an indirect slowing of processing speed due to inattention. Overlapping cognitive effects can also be caused by direct affection of brain areas involved in cognition. 
Since the cognitive effects of depression overlaps with most, if not all of these possible co-factors, it is challenging to distinguish between the origins of the cognitive effects when a patient has a comorbid condition overlapping depression. However, the evidence points to a worsening of perhaps all the cognitive functions affected in depression if any of these co-factors are present.

\subsection{Concluding Remarks}

Information processing speed is a key factor when addressing the effect of aging and depression, and the combination of aging and depression seems to amplify cognitive symptoms. It is a particular function related to attentional ability that is directly affected, while most cognitive functions that require alertness and attention are secondarily affected. Inhibition is one executive function which is particularly associated with depression, where there are independent findings which suggest that this function may be at the core of understanding the nature of depression [13] [18].

Neuropsychological tests are useful in assessing depression in aging, but may not predict depression without access to premorbid information and additional information about the subject. Tests that are particularly sensitive to cognitive delay in depressed elderly patents are identified in the present review. In order to address specific questions, particularly designed laboratory test should be designed, or the combination of cognitive test should be carefully considered to ensure that they address the particular research question, and that other effects may be accounted for. Thus, in group studies, it is important to include tests that are targeting a specific cognitive function or sub-function. Several neuropsychological tests are therefore not appropriate for use in group studies because poor scores on a group level may be due to several different individual subject factors, and should be controlled at the individual level in order to enable prediction to which sub-functions are impaired. A systematic approach to assessing cognitive decline due to depression is needed.

\subsection{Further Work}

Even though attention and inhibition are closely linked cognitive abilities, their relationship should be investigated further. It is not clear whether depression affects inhibition ability directly or if poor inhibition in depression is due to reduced information processing speed and attentional ability because of conflicting negative thought which is a consequence of the depression. This relationship may be studied with fMRI or other imaging techniques in combination with neuropsychological tests or tailored laboratory tests which measure inhibition ability, attention to stimuli and processing speed separately, or include controls for each of these factors. The study groups should be carefully selected so that they are as homogeneous as possible, with respect to depression state and severity, medication, and diagnose, or these factors should be controlled for. Control group needs to be matched for education and age, and perhaps also other co-factors as discussed above.

\section{References}

[1] Bhardwaj, A., Wilkinson, P., Srivastava, C., and Sharma, M. (2010) Cognitive Deficits in Euthymic Patients with Recurrent Depression. The Journal of Nervous and Mental Disease, 198, 513-515. http://dx.doi.org/10.1097/NMD.0b013e3181e4c5ba

[2] McClintok, S.M., Husain, M.M., Greer, T.L. and Cullum, C.M. (2010) Association between Depression Severity and Neurocognitive Function in Major Depressive Disorder: A Review and Synthesis. Neuropsychology, 24, 9-34. http://dx.doi.org/10.1037/a0017336

[3] Story, T.J., Potter, G.G., Attix, D.K., Welsh-Bohmer, K.A. and Steffens, D.C. (2008) Neurocognitive Correlates of Response to Treatment in Late-Life Depression. American Journal of Geriatric Psychiatry, 16, 752-759. http://dx.doi.org/10.1097/JGP.0b013e31817e739a

[4] Crocco, E.A., Castro, K. and Loewenstein, D.A. (2010) How Late-Life Depression Affects Cognition: Neural Mechanisms. Current Psychiatry Reports, 12, 34-38. http://dx.doi.org/10.1007/s11920-009-0081-2

[5] Thomas, A.J., Gallagher, P., Robinson, L.J., Porter, R.J., Young, A.H., Ferrier, I.N. and O’Brien, J.T. (2009) A Comparison of Neurocognitive Impairment in Younger and Older Adults with Major Depression. Psychology and Medicine, 39, 725-733. http://dx.doi.org/10.1017/S0033291708004042

[6] Ptak, R. (2012) The Frontoparietal Attention Network of the Human Brain: Action, Saliency, and the Priority Map of the Environment. Neuroscientist, 18, 502-515. http://dx.doi.org/10.1177/1073858411409051

[7] Hasher, L. and Zacks, R.T. (1979) Automatic and Effortful Processes in Memory. Journal of Experimental Psychology: 
General, 108, 356-388. http://dx.doi.org/10.1037/0096-3445.108.3.356

[8] Hartlage, S., Alloy, L.B., Vazquez, C. and Dykman, B. (1993) Automatic and Effortful Processing in Depression. Psycholical Bulletin, 113, 247-278. http://dx.doi.org/10.1037/0033-2909.113.2.247

[9] Bush, G. (2010) Attention-Deficit/Hyperactivity Disorder and Attention Networks. Neuropsycho-Pharmacology, 35, 278-300. http://dx.doi.org/10.1038/npp.2009.120

[10] Bush, G. and Shin, L.M. (2006) The Multi-Source Interference Task: An fMRI Task That Reliably Activates the Cingulo-Frontal-Parietal Cognitive/Attention Network. Nature Protocols, 1, 308-313. http://dx.doi.org/10.1038/nprot.2006.48

[11] Naghavi, H.R. and Nyberg, L. (2005) Common Fronto-Parietal Activity in Attention, Memory, and Consciousness: Shared Demands on Integration? Conscious Cognition, 14, 390-425. http://dx.doi.org/10.1016/j.concog.2004.10.003

[12] Albinet, C.T., Boucard, G., Bouquet, C.A. and Audiffren, M. (2012) Processing Speed and Executive Functions in Cognitive Aging: How to Disentangle Their Mutual Relationship? Brain and Cognition, 79, 1-11. http://dx.doi.org/10.1016/j.bandc.2012.02.001

[13] Roche, R.A.P., Garavan, H., Foxe, J.J. and O’Mara, S.M. (2005) Individual Differences Discriminate Event-Related Potentials But Not Performance during Response Inhibition. Experimental Brain Research, 160, 60-70.

http://dx.doi.org/10.1007/s00221-004-1985-Z

[14] Jungwirth, S., Zehetmayer, S., Hinterberger, M., Kudronovsky-Moser, S., Weissgram, S., Traglm K.H. and Fisher, P. (2011) The Influence of Depression on Processing Speed and Executive Function in Nondemented Subjects Aged 75. Journal of the International Neuropsychological Society, 17, 822-831. http://dx.doi.org/10.1017/S135561771100083X

[15] Krishnan, K.R., Taylor, W.D., McQuoid, D.R., McFall, J.R., Payne, M.E., Provenzale, J.M. and Steffens, D.C. (2004) Clinical Characteristics of Magnetic Resonance Imaging-Defined Subcortical Ischemic Depression. Biological Psychiatry, 23, 519-522. http://dx.doi.org/10.1016/0006-3223(88)90025-X

[16] Hugdahl, K., Specht, K., Biringer, E., Weis, S., Elliott, R., Hammar, Å., Ersland, L. and Lund, A. (2007) Increased Parietal and Froontal Activation after Remission from Recurrent Major Depression: A Repeated fMRI Study. Cognitive Therapy and Research, 31, 147-160. http://dx.doi.org/10.1007/s10608-006-9116-8

[17] Peterson, B.S., Warner, V., Bansal, R., Zhu, H., Hao, X., Liu, J., Durkin, K., Adams, P.B., Wickramaratne, P. and Weissman, M.M. (2009) Cortical Thinning in Persons at Increased Familiar Risk for Major Depression. Proceedings of the National Academy of Science, 106, 6273-6278. http://dx.doi.org/10.1073/pnas.0805311106

[18] Katz, R., De Sanctis, P., Mahoney, J.R., Sehatpour, P., Murphy, C.F., Gomez-Ramirez, M., Alexopoulos, G.S. and Foxe, J.J. (2010) Cognitive Control in Late-Life Depression: Response Inhibition Deficits and Dysfunction of the Anterior Cingulate Cortex. American Journal of Geriatric Psychiatry, 18, 1017-1025. http://dx.doi.org/10.1097/JGP.0b013e3181d695f2

[19] Salthouse, T.A. (1996) The Processing-Speed Theory of Adult Age Differences in Cognition. Psychological Review, 103, 403-428. http://dx.doi.org/10.1037/0033-295X.103.3.403

[20] Salthouse, T.A. (2012) Consequences of Age-Related Cognitive Declines. Annual Review of Psychology, 63, $201-226$. http://dx.doi.org/10.1146/annurev-psych-120710-100328

[21] Butters, M.A., Whyte, E.M., Nebes, R.D., Begley, M.A., Dew, M.A., Mulsant, B.H., Zmuda, M.D., Bhalla, R., Maltzer, C.C., Pollock, B.G., Reynolds III, C.F. and Becker, J.T. (2004) The Nature and Determinants of Neuropsychological Functioning in Late-Life Depression. JAMA Psychiatry, 61, 587-595. http://dx.doi.org/10.1001/archpsyc.61.6.587

[22] Baune, B.T., Suslow, T., Engelien, A., Arolt, V. and Berger, K. (2006) The Association between Depressive Mood and Cognitive Performance in an Elderly General Population-The MEMO Study. Dementia and Geriatric Cognitive Disorders, 22, 142-149. http://dx.doi.org/10.1159/000093745

[23] van den Kommer, T.N., Comijs, H.C., Aartsen, M.J., Huisman, M., Deeg, D.J. and Beekman, A.T. (2013) Depression and Cognition: How Do They Interrelate in Old Age? American Journal of Geriatric Psychiatry, 21, 398-410. http://dx.doi.org/10.1016/j.jagp.2012.12.015

[24] El Haj, M. and Allain, P. (2012) What Do We Know about the Relationship between Source Monitoring Deficits and Executive Dysfunction? Neuropsychological Rehabilitation, 22, 449-472. http://dx.doi.org/10.1080/09602011.2012.658267

[25] Koster, E.H.W., De Lissnyder, E., Derakshan, N. and De Raedt, R. (2011) Understanding Depressive Rumination from a Cognitive Science Perspective: The Impaired Disengagement Hypothesis. Clinical Psychology Review, 31, 138-145. http://dx.doi.org/10.1016/j.cpr.2010.08.005

[26] Fonseca, R.P., Zimmermann, N., Scherer, L.C., de Mattos Pimenta Parente, M.A. and Ska, B. (2010) Episodic Memory, Concentrated Attention and Processing Speed in Aging, a Comparative Study of Brazilian Age Groups. Dementia \& 
Neuropsychologia, 4, 91-97.

[27] Farrin, L., Hull, L., Unwin, C., Wykes, T. and David, A. (2003) Effects of Depressed Mood on Objective and Subjective Measures of Attention. Journal of Neuropsychiatry \& Clinical Neurosciences, 15, 98-104. http://dx.doi.org/10.1176/appi.neuropsych.15.1.98

[28] Morimoto, S.S., Gunning, F.M., Murphy, C.F., Kanellopoulos, D., Kelly Jr., R.E. and Alexopoulos, G.S. (2011) Executive Function and Short-Term Remission of Geriatric Depression: The Role of Semantic Strategy. American Journal of Geriatric Psychiatry, 19, 115-122. http://dx.doi.org/10.1097/JGP.0b013e3181e751c4

[29] Carlson, M.C., Fried, L.P., Xue, L.P., Bandeen-Roche, K., Zeger, S.L. and Brandt, J. (1999) Association between Executive Attention and Physical Functional Performance in Community-Dwelling Older Women. Journal of Gerontology, British Psychological Society of Science, 54, 262-270.

[30] Royall, D.R., Palmer, R., Chiodo, L.K. and Polk, M.J. (2012) Depressive Symptoms Predict Longitudinal Change in Executive Control but Not Memory. International Journal of Geriatric Psychiatry, 27, 89-96. http://dx.doi.org/10.1002/gps.2697

[31] Paelecke-Habermann, Y., Pohl, J. and Leplow, B. (2005) Attention and Executive Functions in Remitted Major Depression Patients. Journal of Affective Disorders, 89, 125-135. http://dx.doi.org/10.1016/j.jad.2005.09.006

[32] Reppermund, S., Brodaty, H., Crawford, J.D., Kochan, N.A., Slavin, M.J., Trollor, J.N., Draper, B. and Sachdev, P.S. (2011) The Relationship of Current Depressive Symptoms and Past Depression with Cognitive Impairment and Instrumental Activities of Daily Living in an Elderly Population: The Sydney Memory and Ageing Study. Journal of Psychiatric Research, 45, 1600-1607. http://dx.doi.org/10.1016/j.jpsychires.2011.08.001

[33] Turatto, M., Benso, F., Facoetti, A., Galfano, G., Mascetti, G.G. and Umiltá, C. (2000) Automatic and Voluntary Focusing of Attention. Perception \& Psychophysics, 62, 935-952. http://dx.doi.org/10.3758/BF03212079

[34] Yantis, S. and Jonides, J. (1990) Abrupt Visual Onset and Selective Attention: Voluntary vs. Automatic Allocation. Journal of Experimental Psychology: Human Perception \& Performance, 16, 121-134. http://dx.doi.org/10.1037/0096-1523.16.1.121

[35] Schneider, W. and Fisk, A.D. (1982) Concurrent Automatic and Controlled Visual Search: Can Processing Occur without Resource Cost? Journal of Experimental Psychology: Learning, Memory, and Cognition, 8, 261-278.

[36] Massman, P.J., Delis, D.C., Butters, N., Dupont, R.M. and Gillin, J.C. (1992) The Subcortical Dysfunction Hypothesis of Memory Deficits in Depression: Neuropsychological Validation in a Subgroup of Patients. Journal of Clinical and Experimental Neuropsychology, 14, 687-706. http://dx.doi.org/10.1080/01688639208402856

[37] Veiel, H.O. (1997) A Preliminary Profile of Neuropsychological Deficits Associated with Major Depression. Journal of Clinical and Experimental Neuropsychology, 19, 587-603. http://dx.doi.org/10.1080/01688639708403745

[38] Weingartner, H., Cohen, R.M. and Murphey, D.L. (1981) Cognitive Processes in Depression. JAMA Psychiatry, 38, 42-47. http://dx.doi.org/10.1001/archpsyc.1981.01780260044004

[39] Fisk, A.D. and Schneider, W. (1981) Control and Automatic Processing during Tasks Requiring Sustained Attention: A New Approach to Vigilance. Human Factors, 23, 737-750.

[40] Royall, D.R., Cordes, J.A., Velez, A., Edwards, A. and Schillerstrom, J.S. (2009) Sertraline Improves Executive Function in Patients with Vascular Cognitive Impairment. Journal of Neuropsychiatry and Clinical Neuroscience, 21, 445-454. http://dx.doi.org/10.1176/appi.neuropsych.21.4.445

[41] Boyle, L.L., Lyness, J.M., Duberstein, P.R., Karuza, J., King, D.A., Messing, S. and Tu, X. (2010) Trait Neuroticism, Depression, and Cognitive Function in Older Primary Care Patients. American Journal of Geriatric Psychiatry, 18, 305-312. http://dx.doi.org/10.1097/JGP.0b013e3181c2941b

[42] Halstead, W.C. (1947) Brain and Intelligence: A Quantitative Study of the Frontal Lobes. University of Chicago Press, Chicago.

[43] Rosvold, H.E., Mirsky, A.F., Sarason, I., Bransome, E.D. and Beck, L.H. (1956) A Continuous Performance Test of Brain Damage. Journal of Consulting Psychology, 20, 343-350. http://dx.doi.org/10.1037/h0043220

[44] Robertson, I.H., Manly, T. and Andrade, J. (1997) Oops! Performance Correlates of Everyday Attentional Failures in Traumatic Brain Injured and Normal Subjects. Neuropsychologia, 35, 747-758. http://dx.doi.org/10.1016/S0028-3932(97)00015-8

[45] Berardi, A., Parasuraman, R. and Haxby, J. (2001) Overall Vigilance and Sustained Attention Decrements in Healthy Aging. Experimental Aging Research, 27, 19-39. http://dx.doi.org/10.1080/036107301750046124

[46] Moruzzi, G. and Magoun, H.W. (1949) Brain Stem Reticular Formation and Activation of the EEG. Electroencephalography and Clinical Neurophysiology, 1, 455-473. http://dx.doi.org/10.1016/0013-4694(49)90219-9

[47] Moore, H., Sarter, M. and Bruno, J.P. (1993) Bidirectional Modulation of Stimulated Cortical Acetylcholine Release by Benzodiazepine Receptor Ligands. Brain Research, 596, 17-29. http://dx.doi.org/10.1016/0006-8993(92)91527-L 
[48] Janowsky, D.S., El-Yousef, M.K. and Davis, J.M. (1974) Acetylcholine and Depression. Psychosomatic Medicine, 36, 248-257.

[49] Cherry, E.C. (1953) Some Experiments on the Recognition of Speech, with One and with Two Ears. Journal of the Acoustical Society of America, 25, 974-979. http://dx.doi.org/10.1121/1.1907229

[50] Hugdahl, K., Løberg, E.M., Specht, K., Steen, V.M., van-Wageningen, H. and Jørgensen, H.A. (2008) Auditory Hallucinations in Schizophrenia: The Role of Cognitive, Brain Structural and Genetic Disturbances in the Left Temporal Lobe. Frontiers of Human Neuroscience, 1, 1-10.

[51] Wale, J. and Carr, V. (1990) Differences in Dichotic Listening Asymmetries in Depression According to Symptomatology. Journal of Affective Disorders, 18, 1-9. http://dx.doi.org/10.1016/0165-0327(90)90111-K

[52] Okonkwo, O.C., Wadley, V.G., Ball, K., Vance, D.E. and Crowe, M. (2008) Dissociations in Visual Attention Deficits among Persons with Mild Cognitive Impairment. Aging, Neuropsychology, and Cognition: A Journal on Normal and Dysfunctional Development, 15, 492-505.

[53] Hammar, A., Lund, A. and Hugdahl, K. (2003) Long-Lasting Cognitive Impairment in Unipolar Major Depression: A 6-Month Follow-Up Study. Psychiatry Research, 118, 189-196. http://dx.doi.org/10.1016/S0165-1781(03)00075-1

[54] Hamilton, M. (1960) A Rating Scale for Depression. Journal of Neurology, Neurosurgery and Psychiatry, $23,56-62$. http://dx.doi.org/10.1136/jnnp.23.1.56

[55] Majer, M., Ising, M., Künzel, H., Binder, E.B., Holsboer, F., Modell, S. and Zihl, J. (2004) Impaired Divided Attention Predicts Delayed Response and Risk to Relapse in Subjects with Depressive Disorders. Psychological Medicine, 34, 1453-1463. http://dx.doi.org/10.1017/S0033291704002697

[56] Elderkin-Thompson, V., Kumar, A., Mintz, J., Boone, K., Bahng, E. and Lavretsky, H. (2004) Executive Dysfunction and Visuospatial Ability among Depressed Elders in a Community Setting. Archives of Clinical Neuropsychology, 19, 597-611. http://dx.doi.org/10.1016/j.acn.2003.08.009

[57] Lamberty, G.J. and Biellauskas, L.A. (1993) Distinguishing between Depression and Dementia in the Elderly: A Review of Neuropsychological Findings. Archives of Clinical Neuropsychlogy, 8, 149-170. http://dx.doi.org/10.1093/arclin/8.2.149

[58] Beaudreau, S.A. and O’Hara, R. (2009) The Association of Anxiety and Depressive Symptoms with Cognitive Performance in Community-Dwelling Older Adults. Psychology and Aging, 24, 507-512. http://dx.doi.org/10.1037/a0016035

[59] MacDonald, K.B. (2008) Effortful Control, Explicit Processing, and the Regulation of Human Evolved Predispositions. Psychological Review, 115, 1012-1031. http://dx.doi.org/10.1037/a0013327

[60] Gardiner, J.M., Gregg, V.H. and Karayianni, I. (2006) Recognition Memory and Awareness: Occurrence of Perceptual Effects in Remembering or in Knowing Depends on Conscious Resources at Encoding, but Not at Retrieval. Memory \& Cognition, 34, 227-239. http://dx.doi.org/10.3758/BF03193401

[61] Dunn, J.C. (2004) Remember-Know: A Matter of Confidence. Psychological Review, 111, 524-542. http://dx.doi.org/10.1037/0033-295X.111.2.524

[62] Yonelinas, A.P. (2002) The Nature of Recollection and Familiarity: A Review of 30 Years of Research. Journal of Memory and Language, 46, 441-517. http://dx.doi.org/10.1006/jmla.2002.2864

[63] Drakeford, J.L., Edelstyn, N.M., Oyebode, F., Srivastava, S., Calthorpe, W.R. and Mukherjee, T. (2010) Recollection Deficiencies in Patients with Major Depressive Disorder. Psychiatry Research, 175, 205-210. http://dx.doi.org/10.1016/j.psychres.2008.08.010

[64] Eysenck, M.W., Derakshan, N., Santos, R. and Clavo, M.G. (2007) Anxiety and Cognitive Performance: Attentional Control Theory. Emotion, 7, 336-353. http://dx.doi.org/10.1037/1528-3542.7.2.336

[65] Lamar, M., Charlton, R., Zhang, A. and Kumar, A. (2012) Differential Associations between Types of Verbal Memory and Prefrontal Brain Structure in Healthy Aging and Late Life Depression. Neuropsychologia, 50, 1823-1829. http://dx.doi.org/10.1016/j.neuropsychologia.2012.04.007

[66] Tullis, J.G. and Benjamin, A.S. (2012) Consequences of Restudy Choices in Younger and Older Learners. Psychonomic Bulletin and Review, 19, 743-749. http://dx.doi.org/10.3758/s13423-012-0266-2

[67] Milner, B. (1963) Effect of Different Brain Lesions on Card Sorting. JAMA Neurology, 9, 90-100. http://dx.doi.org/10.1001/archneur.1963.00460070100010

[68] Heaton, R.K. (1981) A manual for the Wisconsin Card Sorting Test. Psychological Assessment Resources, Odessa.

[69] Miyake, A., Friedman, N.P., Emerson, M.J., Witzki, A.H. and Howerter, A. (2000) The Unity and Diversity of Executive Functions and Their Contribution to Complex 'Frontal Lobe' Tasks: A Latent Variable Analysis. Cognitive Psychology, 41, 49-100. http://dx.doi.org/10.1006/cogp.1999.0734

[70] Kim, Y.R., Kim, J.E. and Kim, M.H. (2010) Impaired Set-Shifting Ability in Patients with Eating Disorders, Which Is Not Moderated by Their Catechol-O-Methyltransferase Val158Met Genotype. Psychiatry Investigation, 7, $298-301$. 
http://dx.doi.org/10.4306/pi.2010.7.4.298

[71] Roberts, M.E., Tchanturia, K., Stahl, D., Southgate, L. and Treasure, J. (2007) A Systematic Review and Meta-Analysis of Set-Shifting Ability in Eating Disorders. Psychological Medicine, 37, 1075-1084. http://dx.doi.org/10.1017/S0033291707009877

[72] Armstrong, T. and Olatunji, B.O. (2012) Eye Tracking of Attention in the Affective Disorders: A Meta-Analytic Review and Synthesis. Clinical Psychology Review, 32, 704-723. http://dx.doi.org/10.1016/j.cpr.2012.09.004

[73] Stroop, J.R. (1935) Studies of Interference in Serial Verbal Reactions. Journal of Experimental Psychology, 18, 643662. http://dx.doi.org/10.1037/h0054651

[74] Benton, A.L. (1968) Differential Behavioral Effects in Frontal Lobe Disease. Neuropsychologia, 6, 53-60. http://dx.doi.org/10.1016/0028-3932(68)90038-9

[75] O’Bryant, S.E., Hall, J.R., Cukrowicz, K.C., Edwards, M., Johnson, L.A., Lefforge, D., Jenkins, M. and Dentino, A. (2011) The Differential Impact of Depressive Symptom Clusters on Cognition in a Rural Multi-Ethnic Cohort: A Project FRONTIER Study. International Journal of Geriatric Psychiatry, 26, 199-205. http://dx.doi.org/10.1002/gps.2514

[76] Hawkins, K.A., Sledge, W.H., Orleans, J.F., Quinlan, D.M., Rakfeldt, J. and Hoffman, R.E. (1995) Can Digit SymbolVerbal Fluency Comparisons Facilitate Detection of Pseudodementia? A Preliminary Study. European Archives of Psychiatry and Clinical Neuroscience, 244, 317-319. http://dx.doi.org/10.1007/BF02190410

[77] Johnson, M.K., Hashtroudi, S. and Lindsay, D.S. (1993) Source Monitoring. Psychological Bulletin, 114, 3-28. http://dx.doi.org/10.1037/0033-2909.114.1.3

[78] El Haj, M. and Allain, P. (2012) Relationship between Source Monitoring in Episodic Memory and Executive Function in Normal Aging. Gériatrie et Psychologie Neuropsychiatrie du Vieillissement, 10, 197-205. (article in French).

[79] von Hecker, U. and Meiser, T. (2005) Defocused Attention in Depressed Mood: Evidence from Source Monitoring. Emotion, 5, 456-463. http://dx.doi.org/10.1037/1528-3542.5.4.456

[80] Beck, A.T., Ward, C.H., Mendelson, M., Mock, J. and Erbaugh, J. (1961) An Inventory for Measuring Depression. JAMA Psychiatry, 4, 561-571. http://dx.doi.org/10.1001/archpsyc.1961.01710120031004

[81] Ito, S., Stuphorn, V., Brown, J.W. and Schall, J.D. (2003) Performance Monitoring by the Anterior Cingulate Cortex during Saccade Countermanding. Science, 302, 120-122. http://dx.doi.org/10.1126/science.1087847

[82] Holroyd, C.B., Nieuwenhuis, S., Mars, R.B. and Coles, M.G.H. (2004) Anterior Cingulate Cortex, Selection for Action, and Error Processing. In: Posner, M.I., Ed., Cognitive Neuroscience of Attention, Guilford Press, New York, 219-231.

[83] Hester, R., Foxe, J.J., Molholm, S., Shpaner, M. and Garavan, H. (2005) Neural Mechanisms Involved in Error Processing: A Comparison of Errors Made with and without Awareness. NeuroImage, 27, 602-608. http://dx.doi.org/10.1016/j.neuroimage.2005.04.035

[84] Botvinick, M.M., Cohen, J.D. and Carter, C.S. (2004) Conflict Monitoring and Anterior Cingulate Cortex: An Update. Trends in Cognitive Sciences, 8, 539-546. http://dx.doi.org/10.1016/j.tics.2004.10.003

[85] Holmes, A.J. and Pizzagalli, D.A. (2008) Response Conflict and Frontocingulate Dysfunction in Unmedicated Participants with Major Depression. Neuropsychologia, 46, 2904-2913.

http://dx.doi.org/10.1016/j.neuropsychologia.2008.05.028

[86] Bartholow, B.D., Henry, E.A., Lust, S.A., Saults, J.S. and Wood, P.K. (2012) Alcohol Effects on Performance Monitoring and Adjustment: Affect Modulation and Impairment of Evaluative Cognitive Control. Journal of Abnormal Psychology, 121, 173-186. http://dx.doi.org/10.1037/a0023664

[87] Inzlicht, M. and Al-Khindi, T. (2012) ERN and the Placebo: A Misattribution Approach to Studying the Arousal Properties of the Error-Related Negativity. Journal of Experimental Psychology: General, 141, 799-807. http://dx.doi.org/10.1037/a0027586

[88] Georgiadi, E., Liotti, M., Nixon, N.L. and Liddle, P.F. (2011) Electrophysiological Evidence for Abnormal Error Monitoring in Recurrent Major Depressive Disorder. Psychophysiology, 48, 1192-1202. http://dx.doi.org/10.1111/j.1469-8986.2011.01198.x

[89] MacLeod, C.M., Dodd, M.D., Sheard, E.D., Wilson, D.E. and Bibi, U. (2003) In Opposition to Inhibition. Psychology of Learning and Motivation, 43, 163-214. http://dx.doi.org/10.1016/S0079-7421(03)01014-4

[90] Luria, A.R. and Homskaya, E.D. (1964) Disturbance in the Regulative Role of Speech with Frontal Lobe Lesions. In: Akert, W.A., Ed., The Frontal Granular Cortex and Behavior, McGraw Hill, New York, 353-371.

[91] Fossati, P., Ergis, A.M. and Allilaire, J.F. (2002) Executive Functioning in Unipolar Depression: A Review. Encephale, 28, 97-107.

[92] Kaiser, S., Unger, J., Kiefer, M., Markela, J., Mundt, C. and Weisbrod, M. (2003) Executive Control Deficit in Depression: Event-Related Potentials in a Go/Nogo Task. Psychiatry Research, 122, 169-184. 
http://dx.doi.org/10.1016/S0925-4927(03)00004-0

[93] Alexopoulos, G.S., Murphy, C.F., Gunning-Dixon, F.M., Kalayam, B., Katz, R., Kanellopoulos, D., Etwaroo, G.R., Klimstra, S. and Foxe, J.J. (2007) Event-Related Potentials in an Emotional Go/No-Go Task and Remission of Geriatric Depression. Neuroreport, 18, 217-221. http://dx.doi.org/10.1097/WNR.0b013e328013ceda

[94] De Lissnyder, E., Koster, E.H.W., Derakshan, N. and De Raedt, R. (2010) The Association between Depressive Symptoms and Executive Control Impairments in Response to Emotional and Non-Emotional Information. Cognition and Emotion, 24, 264-280. http://dx.doi.org/10.1080/02699930903378354

[95] Medalla, M. and Barbas, H. (2012) The Anterior Cingulate Cortex May Enhance Inhibition of Lateral Prefrontal Cortex via m2 Cholinergic Receptors at Dual Synaptic Sites. Journal of Neuroscience, 32, 15611-15625. http://dx.doi.org/10.1523/JNEUROSCI.2339-12.2012

[96] Gunning, F.M., Cheng, J., Murphy, C.F., Kanellopoulos, D., Acuna, J., Hoptman, M.J., Klimstra, S., Morimoto, S., Weinberg, J. and Alexopoulos, G.S. (2009) Anterior Cingulate Cortical Volumes and Treatment Remission of Geriatric Depression. International Journal of Geriatric Psychiatry, 24, 829-836. http://dx.doi.org/10.1002/gps.2290

[97] Wilson, B.A., Alderman, N., Burgess, P.W., Emslie, H. and Evans, J.J. (1996) Behavioural Assessment of the Dysexecutive Syndrome. Thames Valley Test Company, Bury St. Edmunds.

[98] Allain, P., Nicoleau, S., Pinon, K., Etcharry-Bouyx, F., Barré, J., Berrut, G., Dubas, F. and Le Gall, D. (2005) Executive Functioning in Normal Aging: A Study of Action Planning Using the Zoo Map Test. Brain and Cognition, 57, 4-7. http://dx.doi.org/10.1016/j.bandc.2004.08.011

[99] Oosterman, J.M., Wijers, M. and Kessels, R.P.C. (2013) Planning or Something Else? Examining Neuropsychological Predictors of Zoo Map Performance. Applied Neuropsychology: Adult, 20, 103-109. http://dx.doi.org/10.1080/09084282.2012.670150

[100] Murphy, C.F. and Alexopoulos, G.S. (2006) Attention Network Dysfunction and Treatment Response of Geriatric Depression. Journal of Clinical and Experimental Neuropsychology, 28, 96-100. http://dx.doi.org/10.1080/13803390490918101

[101] Montgomery, S.A. and Asberg, M. (1979) A New Depression Scale Designed to be Sensitive to Change. The British Journal of Psychiatry, 134, 382-389. http://dx.doi.org/10.1192/bjp.134.4.382

[102] Engedal, K., Kvaal, K., Korsnes, M., Lage Barca, M., Borza, T., Selbaek, G. and Aakhus, E. (2012) The Validity of the Montgomery-Aasberg Depression Rating Scale as a Screening Tool for Depression in Later Life. Journal of Affective Disorders, 141, 227-232. http://dx.doi.org/10.1016/j.jad.2012.02.042

[103] Biringer, E., Mykletun, A., Dahl, A.A., Smith, A.D., Engedal, K., Nygaard, H.A. and Lund, A. (2005) The Association between Depression, Anxiety, and Cognitive Function in the Elderly General Population, the Hordaland Health Study. International Journal of Geriatric Psychiatry, 20, 989-997. http://dx.doi.org/10.1002/gps.1390

[104] Butters, M.A., Bhalla, R.K., Andreescu, C., Wetherell, J.L., Mantella, R., Begley, A.E. and Lenze, E.J. (2011) Changes in Neuropsychological Functioning Following Treatment for Late-Life Generalised Anxiety Disorder. The British Journal of Psychiatry, 199, 211-218. http://dx.doi.org/10.1192/bjp.bp.110.090217

[105] Kessler, R.C., Berglund, P., Demler, O., Jin, R., Koretz, D., Merikangaz, K.R., Rush, A.J., Walters, E.E. and Wang, P.S. (2003) The Epidemiology of Major Depressive Disorder: Results from the National Comorbidity Survey Replication (NCS-R). Journal of the American Medical Association, 289, 3095-3105.

http://dx.doi.org/10.1001/jama.289.23.3095 